\title{
Estimating influenza-related sick leave in Norway: Was work absenteeism higher during the 2009 A(H1N1) pandemic compared to seasonal epidemics?
}

B F de Blasio (birgitte.deblasio@medisin.uio.no) ${ }^{1,2}$, Y Xue ${ }^{1,2}$, B Iversen², J Michael Gran ${ }^{1,2}$

1. Department of Biostatistics, Institute of Basic Medical Sciences, University of Oslo, Oslo, Norway

2. Department of Infectious Disease Epidemiology, Division of Infectious Disease Control, Norwegian Institute of Public Health, Oslo, Norway

Citation style for this article:

de Blasio BF, Xue Y, Iversen B, Michael Gran J. Estimating influenza-related sick leave in Norway: Was work absenteeism higher during the $2009 \mathrm{~A}(\mathrm{H} 1 \mathrm{~N} 1)$ pandemic compared to seasonal epidemics?. Euro Surveill. 2012;17(33):pii=20246. Available online: http://www.eurosurveillance.org/ViewArticle.aspx?Articleld=20246

The impact of influenza on work absenteeism is poorly documented. We used data from the national registry and Norway Post AS $(>14,000$ employees) to explore sickness absence patterns from 2005/06 through $2009 / 10$ in Norway. Annually, an estimated $2.868 \%$ (mean $95 \%$ confidence interval (Cl): $2.405-4.820 \%$ ) of the working population obtained sick leave for influenza, of whom $0.915 \%$ (mean $95 \% \mathrm{Cl}$ : $0.453-1.590 \%$ ) had diagnoses for other respiratory illnesses with influenza as underlying cause. In the 2009/10 pandemic season, the absence rate increased 1.5 -fold, mainly due to a $73 \%$ increase in influenza-diagnosed sick leaves. At Norway Post AS, absence related to seasonal influenza accounted for $0.351 \%$ (mean $95 \%$ $\mathrm{Cl}$ : $0.126-0.704 \%)$ of total person-days annually (excluding parental care absence), of which $32-43 \%$ were estimated to be self-certified. Medically certified sick leave increased 1.3-fold in the pandemic season to $0.458 \%$ ( $95 \% \mathrm{Cl}: 0.176-0.856)$, while self-certified sick leave remained at a level typical for seasonal influenza. We found a significant four-fold increase in work loss to care for sick children, $0.048 \%$ (95\% $\mathrm{Cl}: 0.031-0.070 \%)$ of person-days, compared with $0.012 \%$ (95\% Cl: $0.004-0.028 \%$ ) in 2008/09. In conclusion, GP-certified and parental care work absence were higher in the pandemic season. More studies are needed to quantify the burden of self-certified sick leave.

\section{Introduction}

Influenza is a highly contagious disease that affects people of all ages and imposes substantial burdens to the healthcare system. In addition, influenza epidemics result in considerable work absenteeism, and the associated cost of lost productivity is a major component of the financial burden of the disease to society [1-4]. In Europe, the major pandemic in 2009 wave arrived in October to December; in several European countries including Norway it was preceded by a smaller summer wave occurring from July to September. The pandemic was less severe than initially feared: there was immunity among the elderly and the large majority of illnesses were mild and self-limiting [5]. Estimation of influenza-related sick leave is challenging, partly because influenza is rarely laboratory-confirmed and partly because the disease may lead to secondary infection or worsening of underlying chronic illness. Mortality related to influenza is routinely studied, and the most common methods used to study influenza-attributable mortality are: the Serfling method [6], which measures the excess mortality above an expected winter baseline, cyclical regression [7], and Poisson regression informed by influenza surveillance data [8-10]. These approaches have been adopted to estimate excess hospitalisations [11], and more recently to estimate influenza-associated sick leaves [12]. In this study we used Poisson regression because the method can accommodate additional factors, such as accounting for co-circulation of other viruses and adjusting for the unusual timing of the pandemic that occurred in Norway outside the winter period.

We quantified work absence related to the 2009 pandemic in Norway and compared it with work absence for seasonal influenza epidemics, using data from the national registry on general practitioner (GP)-certified sick leave. In Norway, approximately $50 \%$ of the work force has a so-called 'Inclusive Working Life' (IWL) agreement allowing employees the right to full salary during sickness for up to eight calendar days of work absence without a GP certificate. Approximately $40 \%$ of the Norwegian work force has a non-IWL agreement allowing for up to three days of work absence with full salary without a doctor's certificate; the remaining $10 \%$ are self-employed, and this group is not compensated during illness. A considerable part of sickness absence due to influenza is therefore 'self-certified' and unknown to Norwegian public health authorities. In addition, work absence related to caring for sick children is not centrally registered. Previous international studies have found higher influenza-attributable work 
absence among employees living in households with children $[2,13]$

To explore influenza-related work absences for selfcertified sickness and parental care, we further analysed data from the Norwegian postal service (Norway Post AS), a government-owned company and one of the largest firms in Norway with more than 14,000 employees. Because of a monopoly on mail distribution, Norway Post AS is represented in all geographic regions of Norway. The present results provide new insights into the disease burden of influenza, and may serve as reference data in health economic evaluations of influenza.

\section{Methods}

Data

Weekly influenza-like illness (ILI) data were obtained from the Norwegian Institute of Public Health for the period January 2005 to December 2010. The Norwegian GP sentinel network consists of approximately 200 GPs throughout the country and covers approximately $15 \%$ of the population. The influenza surveillance is normally active in the winter season from week 40 to week 20, but was extended in 2009 to year-round reporting due to the on-going pandemic. Sentinel ILI rates are expressed per total number of consultations. The missing ILI levels during the summers of 2005 to 2008 were imputed from the ILI levels in week 20 and week 40 when influenza surveillance ended and started. We assumed that these ILI levels were representative of the baseline ILI activity in periods without influenza epidemics; the levels were all small and comparable (range: $0.47-0.73 \%$ ). In each year separately, weekly ILI levels were found from a linear interpolation between the ILI levels in week 20 and 40.

We used monthly data on all Norwegian laboratory-confirmed respiratory syncytial virus (RSV) cases and norovirus cases from the Department of Virology, Division of Infectious Disease Control, at the Norwegian Institute of Public Health. The data do not convey information on the age of the reported cases. For RSV, smears of secretions from the nasopharynx were collected primarily from hospitalised children with acute respiratory diseases, and the samples analysed using PCR or immunofluorescence. Both the PCR method and the primers varied between laboratories. For norovirus, stool samples were collected mainly from adults during outbreaks in institutions and hospitals (G. Ånestad, personal communication, March 2012) and tested, primarily using in-house $P C R$, but the PCR method and the primers varied between laboratories.Like influenza, both RSV and norovirus are mainly active during the winter months and cause outbreaks involving a substantial proportion of the population, and similar to influenza virus, RSV and norovirus infect the respiratory tract and gastrointestinal epithelial cells, respectively $[14,15]$ Weekly data on the number of individuals who went on sick leave (i.e. counts of new sick leaves) with International Classification of Primary Care (ICPC)-2 diagnoses [16] influenza (R80) and other respiratory diseases (all $R$ diagnoses except R80) were obtained from the Norwegian Labour and Welfare Service (NAV) for the period January 2005 to December 2010. A robust locally weighted regression smoothing (robust LOESS) was applied to the data to eliminate large variations around the New Year and Easter vacations. Monthly employment data during the study period for individuals aged 16 to 74 years were obtained from Statistics Norway [17].

At Norway Post AS, work absence is registered as number of working days lost, whereas the number of employees absent from work is not recorded. We used monthly data on working days lost due to self-certified and GP-certified sick leave from February 2005 to November 2010; data on work absence related to caring for sick children were only available from February 2008 to December 2010.

\section{Regression analyses}

We used log-linear Poisson regression modelling to estimate counts of respiratory sick leaves attributable to influenza in the national sick leave registry data. In the model, weekly numbers of respiratory sick leaves (ICPC-2 all R codes, excluding influenza R80) were explained by ILI rates among 15 to 64 year-olds as a proxy for influenza activity, laboratory-confirmed RSV cases, short- and long-term temporal variables accounting for weekly and seasonal patterns not related to the respiratory infections included, and an interaction term between ILI reporting and the pandemic to account for the fact that the pandemic occurred outside the normal influenza season in Norway:

$$
\begin{aligned}
\hat{S}_{j, s}= & \exp \left[\log \left(S_{j, s}^{\text {tot }}\right)+\beta_{0}+\beta_{1} I L I_{j, s}^{15-64 y}+\sum_{j} \beta_{2, j}^{\text {week }}+\right. \\
& \left.\sum_{s} \beta_{3, s}^{\text {season }}+\beta_{4} R S V_{j, s}+\sum_{k} \beta_{5, k}\left(I L I_{j, s}^{15-64 y} \times I_{k}^{\text {pan }}\right)+\text { error }_{j, s}\right]
\end{aligned}
$$

Here, $\hat{S}_{j, s}$ is the predicted count of new sick leaves in week $j=\{1,2, \ldots 52\}$ and season $\mathrm{s}=\{2004 / 05, \ldots 2010 / 11\}$, $\log \left(S_{j, s}^{\text {tot }}\right)$ is an offset term of the total number of employed persons, $I L I_{j, s}^{15-64 y}$ is the ILI rate in the working population, $\beta_{2, j}^{\text {week }}, \beta_{3, s}^{\text {season }}$ describe the variation within the seasons and between the seasons, respectively, and $R S V_{j, s}$ represents monthly numbers of laboratory-confirmed RSV infections. Lastly, the term $\beta_{5,1}$ describes the interaction between reported ILI activity and the pandemic summer wave using an indicator variable $I_{1}^{p a n}$ that was set equal to one from July to September 2009, and zero otherwise. Similarly the term $\beta_{5,2}$ describes the interaction between ILI activity and the major pandemic wave using an indicator variable $I_{2}^{\text {pan }}$ that was set equal to one from October to December 2009, and zero otherwise. Because the RSV cases were reported on a monthly basis, we created a dummy variable to assign a month-to-week indicator. To account for over-dispersion, we used a quasi-Poisson approach by including a dispersion parameter $\lambda$. We used a similar model to estimate monthly GP-certified and 
Surveillance data for influenza-like illness, respiratory syncytial virus and norovirus, Norway 2005/06-2009/10

A

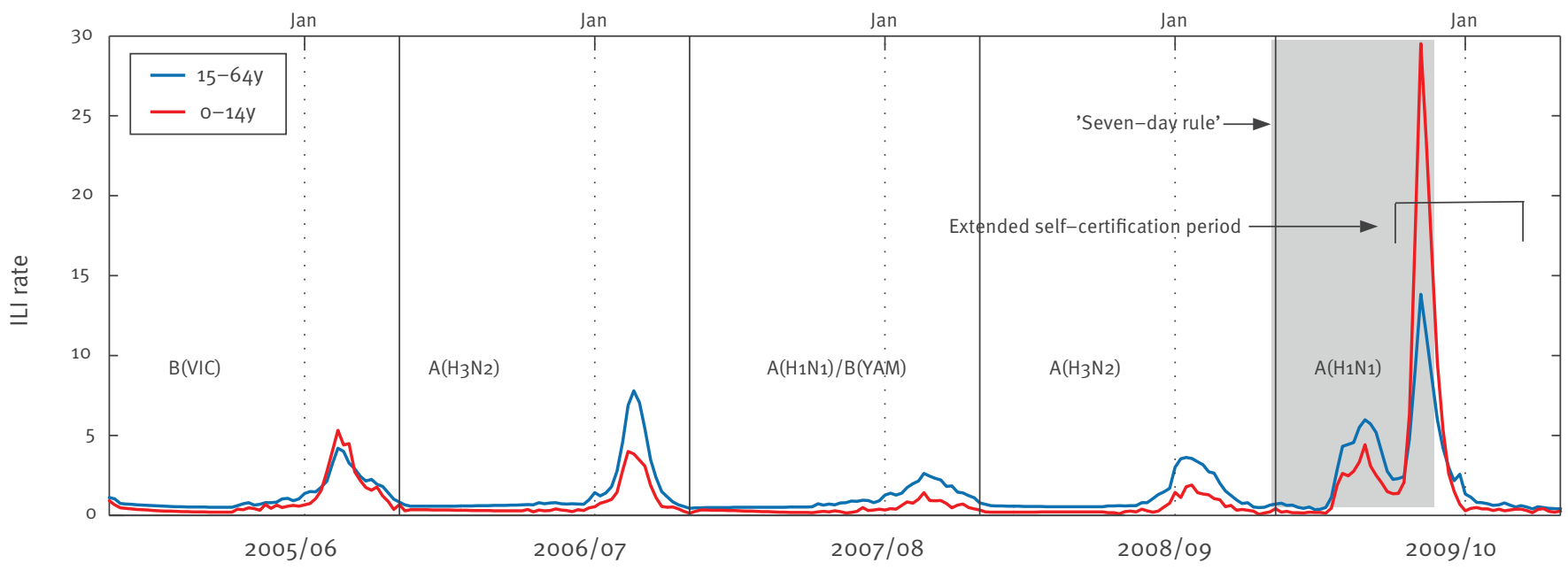

B

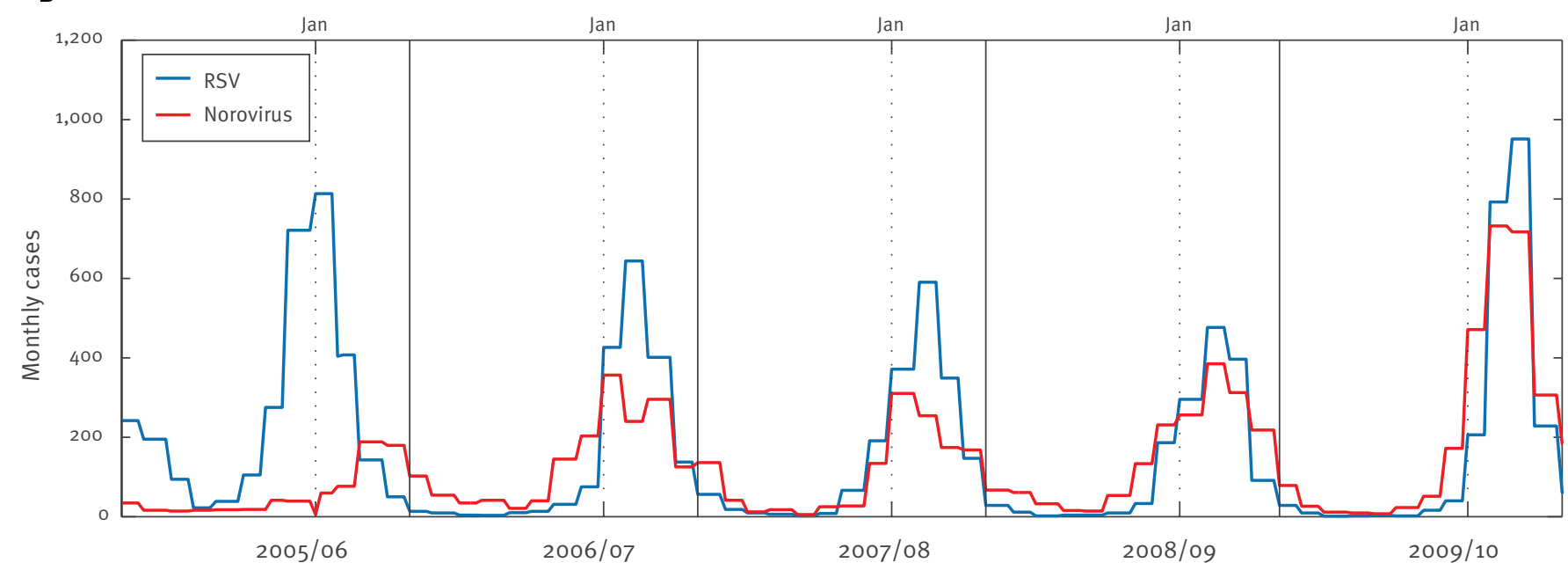

ILI: influenza-like illness; RSV: respiratory syncytial virus.

A: Weekly ILI rates expressed in percentage of total consultations among children and adults. Dominating influenza strains are listed for each season. The time period of extended self-certification from three to eight days is indicated, and the time period during which people e with influenza-like symptoms were recommended to stay at home: seven days after onset of symptoms (gray shaded area).

B: Monthly number of laboratory-confirmed cases of RSV and norovirus infections.

self-certified working days lost at Norway Post AS, except that we included monthly laboratory-confirmed norovirus cases Noro $_{i . s}$, and weekly variables were substituted with monthly predictors $j=\{1,2, \ldots 12\}$. In these models the offset $\log \left(S_{j, s}^{\text {tot }}\right)$ comprised monthly totals of working days.

Norway Post AS started to record data about work absence to care for sick child in 2008. Because observations were sparse, we used a modified Poisson model with few predictor variables to estimate influenza-attributable loss of working days for parents caring for sick children:

$$
\begin{gathered}
\hat{S}_{i}=\exp \left[\log \left(S_{i}^{\text {tot }}\right)+\beta_{0}+\beta_{1} I L I_{i}^{0-14 y}+\beta_{2} \operatorname{Cos}(2 \pi i / 12)+\right. \\
\left.\beta_{3} \operatorname{Sin}(2 \pi i / 12)+\beta_{4} i^{2}+\beta_{5} I^{\text {July }}+\text { error }_{i}\right]
\end{gathered}
$$

Here $\hat{S}_{i}$ is the predicted number of working days lost due to caring for sick children in month $\mathrm{i}=\{1,2, . .34\}$ starting from February 2008, $\log \left(S_{i}^{\text {tot }}\right)$ is the offset term of total monthly working days, and $I L I_{j, s}^{0-14 y}$ is the ILI rate in children. The terms $\operatorname{Cos}(2 \mu i / 12)$ and $\operatorname{Sin}(2 \mu i / 12)$ describe yearly fluctuations in lost working days and $i^{2}$ accounts for the time trend in lost working days (a linear predictor variable was also tested, but it was not significant) and $I^{J u l y}$ is an indicator variable set equal 


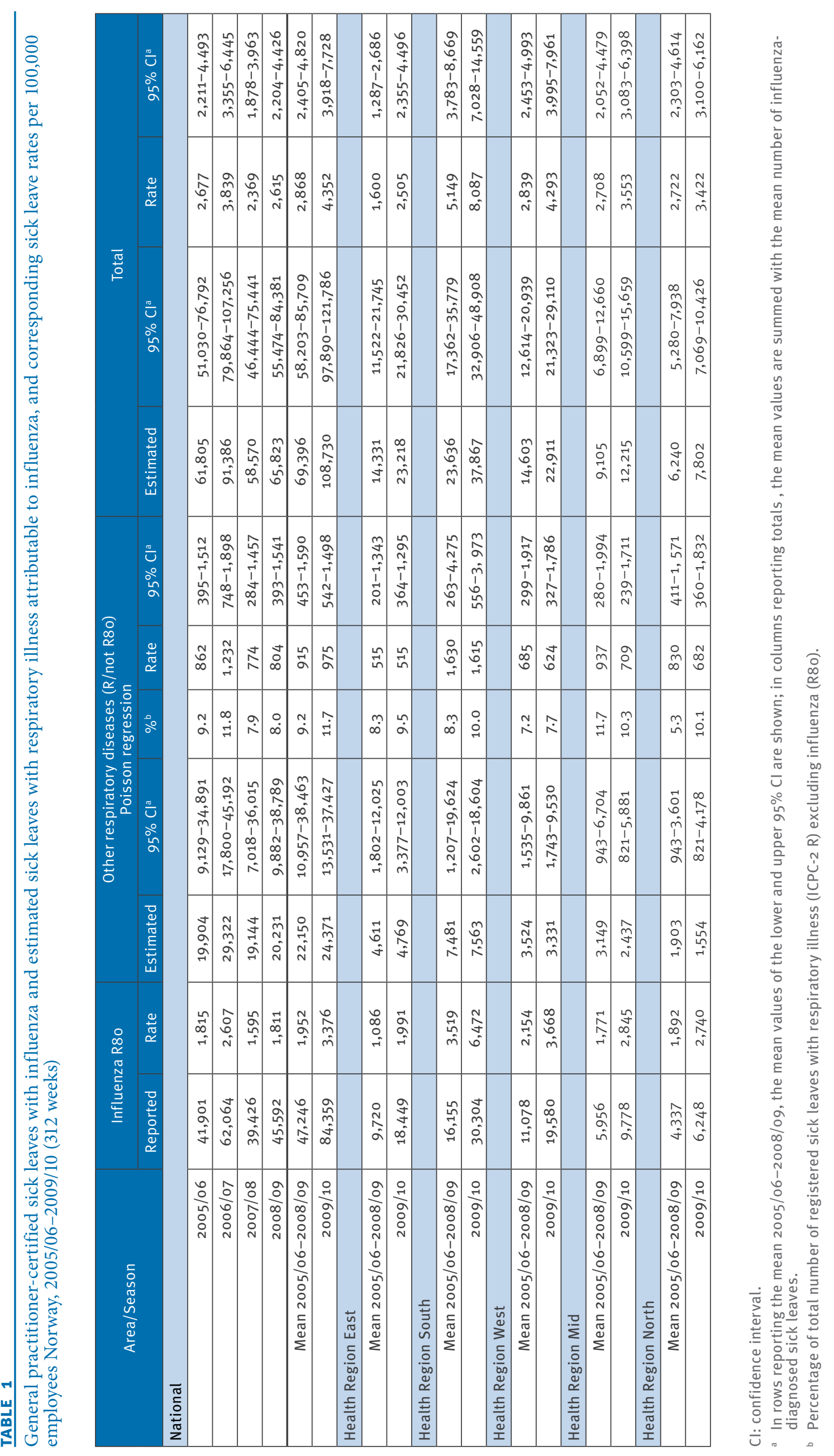


Estimated weekly excess of influenza-attributable general practitioner-certified sick leaves with respiratory illness, Norway, 2005/06-2009/10

A

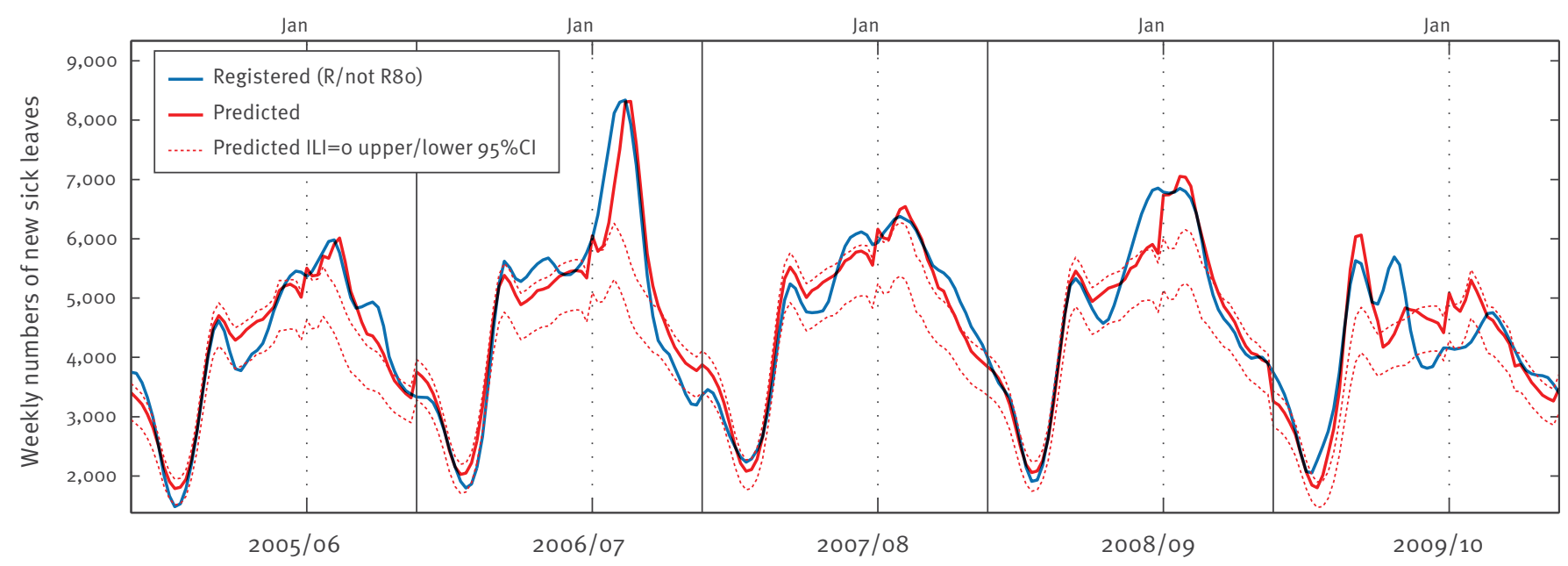

B

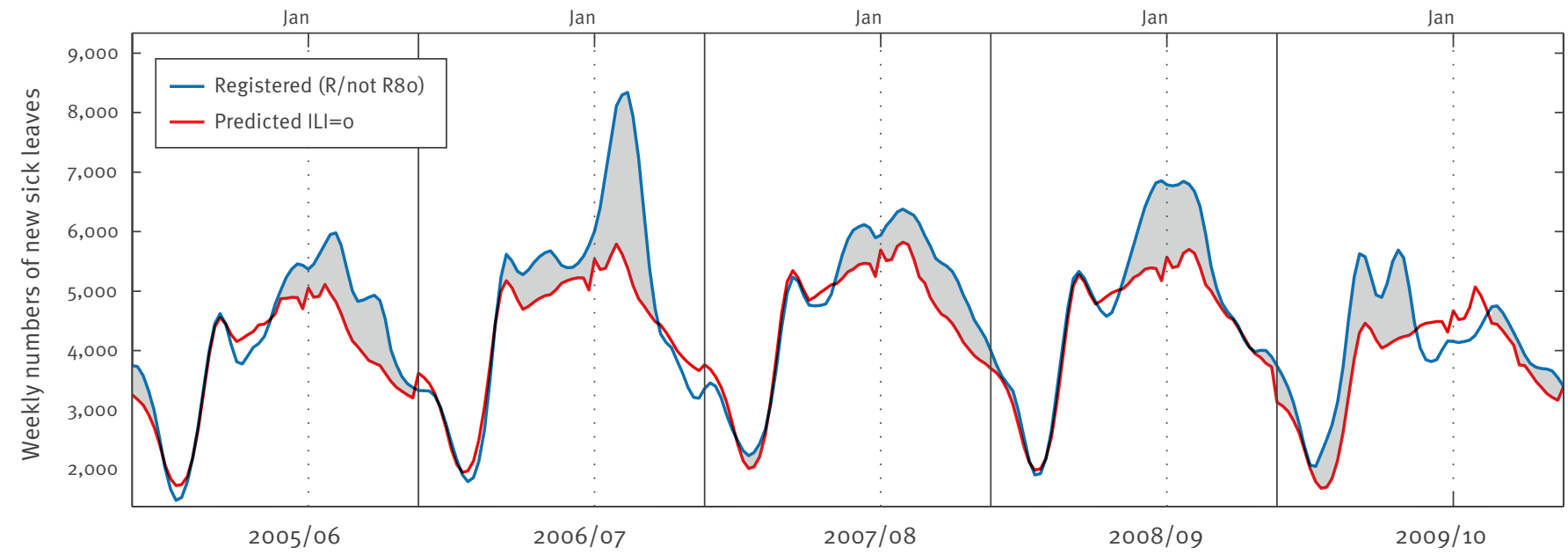

$\mathrm{Cl}$ : confidence interval; ILI: influenza-like illness.

A: Observed (blue) and predicted (red) weekly numbers of respiratory sick leaves; upper and lower $95 \%$ confidence interval of prediction with ILI=0 (dotted red lines).

B: Crude estimation calculated as the difference between observed and predicted (ILI=0); only positive contributions are summed (gray areas).

to 1 in the month of July and zero elsewhere to adjust for low levels of work absence during the main summer holiday period. As above, we adopted a quasi-Poisson approach by fitting a dispersion parameter.

The Poisson regression was conducted using the GLM package in the open source statistical software $R$ version 2.13.0 [18]; data smoothing and graphical plots were done using Matlab version R2011a.

Estimating influenza-associated work absence For each outcome, only significant variables were included in the final models that were used to estimate the sick leave burden. We estimated a Poisson baseline from the number of sick leaves/working days lost for each week/month predicted by the models equation 1.1 and equation 1.2 when influenza activity was set to zero.

A crude estimate of the influenza-associated sick leaves/working days lost for each season was made by subtracting the Poisson baseline at each point in time from the observed values and summing the positive contributions. We considered the uncertainty in the parameter estimates by subtracting the upper and lower $95 \%$ confidence intervals $(\mathrm{Cl})$ of the models from 
TABLE 2

Estimated self-certified work absence and certified work absence at Norway Post AS due to influenza from 2005/06 through 2009/10 (71 months), work absence due to care of sick children from 2008/09 to 2009/10 (34 months)

A

\begin{tabular}{|c|c|c|c|c|c|}
\hline Season & Est. & $95 \% \mathrm{Cl}^{\mathrm{a}}$ & $\%^{\mathrm{b}}$ & Rate $^{c}$ & $95 \% \mathrm{Cl}^{\mathrm{b}}$ \\
\hline \multicolumn{6}{|l|}{ Self-reported } \\
\hline $2005 / 06$ & 4,459 & $2,473-6,434$ & 10.2 & 131 & $72-188$ \\
\hline $2006 / 07$ & 5,208 & $3,175-7,146$ & 12.3 & 157 & $96-215$ \\
\hline $2007 / 08$ & 3,627 & $2,147-5,452$ & 8.4 & 107 & $64-161$ \\
\hline $2008 / 09$ & 4,157 & $2,203-5,919$ & 10.1 & 131 & $70-187$ \\
\hline Mean 2005/06-2008/09 & 4,363 & $2,500-6,238$ & 10.3 & 132 & $75-188$ \\
\hline $2009 / 10$ & 3,695 & $1,841-5,273$ & 9.8 & 127 & $63-181$ \\
\hline \multicolumn{6}{|l|}{ GP-certified } \\
\hline $2005 / 06$ & 9,442 & $2,855-19,427$ & 3.2 & 277 & $84-569$ \\
\hline $2006 / 07$ & 6,877 & $1,221-17,761$ & 2.6 & 207 & $37-536$ \\
\hline $2007 / 08$ & 7,059 & $1,712-16,139$ & 2.8 & 209 & $51-478$ \\
\hline $2008 / 09$ & 5,802 & $1,019-15,488$ & 2.3 & 183 & $32-489$ \\
\hline Mean 2005/06-2008/09 & 7,295 & $1,702-17,204$ & 2.7 & 219 & $51-514$ \\
\hline $2009 / 10$ & 9,640 & $3,286-19,643$ & 4.1 & 331 & $113-675$ \\
\hline \multicolumn{6}{|l|}{ Total Self+GP } \\
\hline Mean 2005/06-2008/09 & 11,658 & $4,202-23,442$ & - & 351 & $126-704$ \\
\hline $2009 / 10$ & 13,335 & $5,126-24,916$ & - & 458 & $176-856$ \\
\hline
\end{tabular}

B

\begin{tabular}{|c|c|c|c|c|c|}
\hline Season & Est. & $95 \% \mathrm{Cl}^{\mathrm{a}}$ & $\%^{\mathrm{b}}$ & Rate $^{c}$ & $95 \% \mathrm{Cl}^{\mathrm{b}}$ \\
\hline \multicolumn{6}{|l|}{ Sick children men } \\
\hline $2008 / 09$ & 228 & $112-502$ & 4.8 & 13 & $6-29$ \\
\hline $2009 / 10$ & 870 & $547-1,251$ & 17.1 & 52 & $33-75$ \\
\hline \multicolumn{6}{|l|}{ Sick children women } \\
\hline $2008 / 09$ & 141 & $26-382$ & 4.0 & 10 & $2-27$ \\
\hline $2009 / 10$ & 539 & $366-774$ & 16.6 & 44 & $30-63$ \\
\hline total $2008 / 09$ & 369 & $138-884$ & 4.5 & 12 & $4-28$ \\
\hline $2009 / 10$ & 1,409 & $913-2,025$ & 16.9 & 48 & $31-70$ \\
\hline \multicolumn{6}{|l|}{ Total Self+GP+children } \\
\hline $2008 / 09$ & 10,328 & $4,339-24,326$ & - & 362 & $131-732$ \\
\hline $2009 / 10$ & 14,743 & $6,040-26,941$ & - & 507 & $208-926$ \\
\hline
\end{tabular}

$\mathrm{Cl}$ : confidence interval; GP: general practitioner.

a In rows reporting the mean 2005/06-2008/09, the mean values of the lower and upper $95 \% \mathrm{Cl}$ are shown;

b Percentage of total number of lost working days (self-certified/GP-certified/sick children).

c Rates of lost working days per 100,000 working days.

the observed values. Again, only those weeks/months with a positive contribution were summed. For consistency between models using weekly and monthly variables, the influenza seasons were defined to last from May to April, although the influenza surveillance (except in 2009/10) ended in week 20 (approximately mid-May).

\section{Results}

Figure 1 shows weekly ILI rates among adults and children, and monthly laboratory-confirmed cases of RSV and norovirus in Norway from 2005/06 through
2009/10. In this period, seasonal influenza epidemics occurred during the months December to April. In 2009, high ILI rates occurred in two separate time periods: a minor summer wave (July to September) and a major wave (October to December). Both RSV and norovirus infections displayed clear seasonal patterns with a peak appearing during the late winter months.

General practitioner-certified sick leaves in national registries associated with influenza The number of GP-certified sick leaves with influenza diagnoses was on average 47,246 in the seasons from 
Estimated monthly excess of self-certified influenza-attributable working days lost at Norway Post AS, 2005/06-2009/10

A

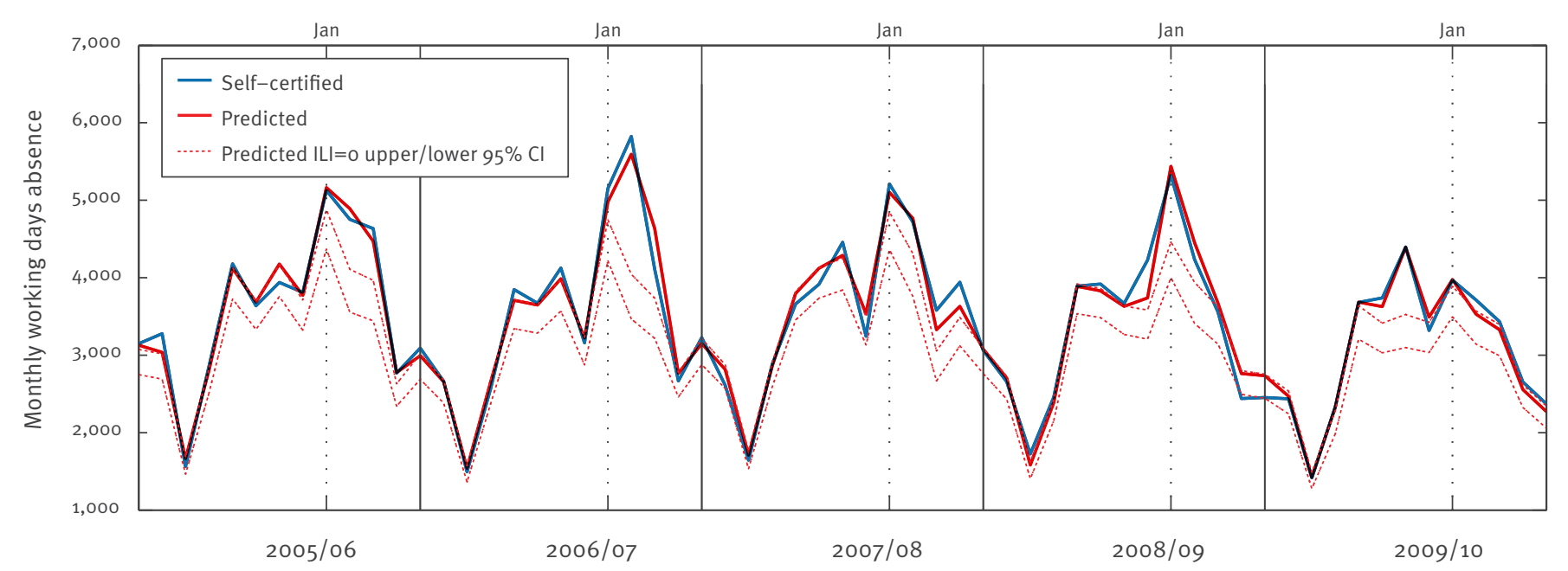

B

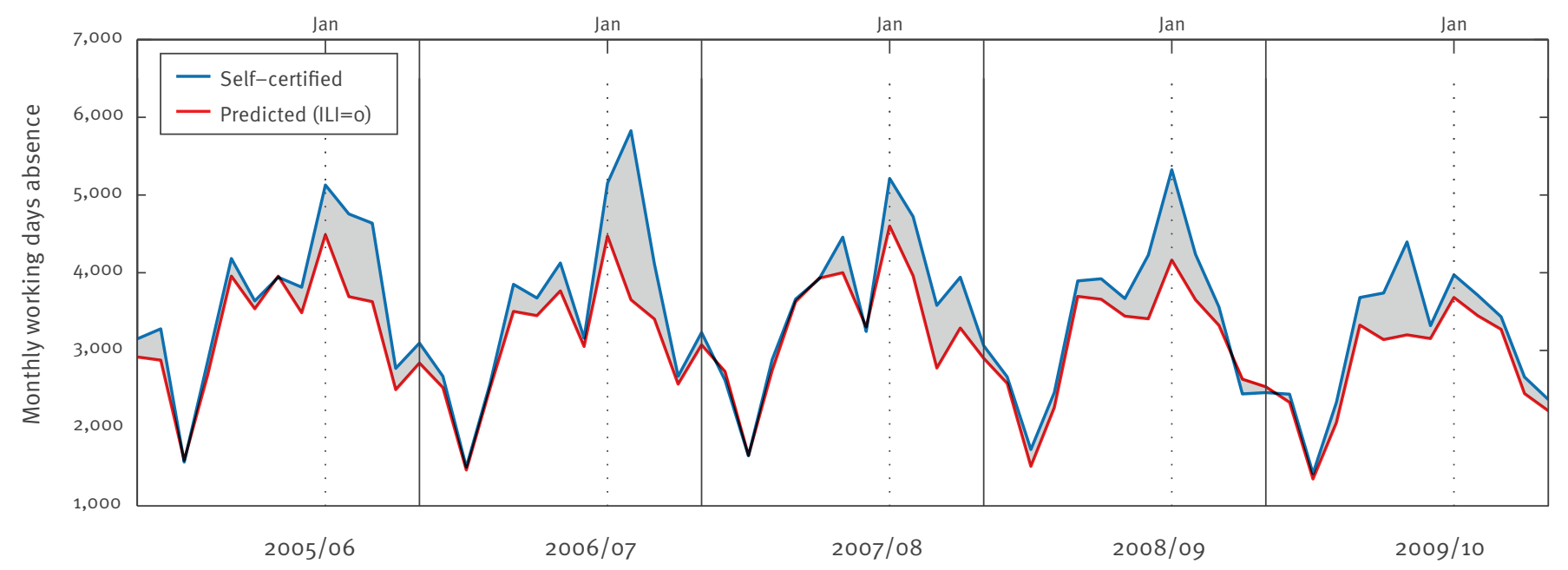

$\mathrm{Cl}$ : confidence interval; ILI: influenza-like illness.

A: Observed (blue) and predicted (red) monthly numbers of self-certified working days lost; upper and lower $95 \%$ confidence interval of prediction with $\mathrm{ILI}=0$ (dotted red lines).

B: Crude estimation calculated as the difference between observed and predicted (ILI=0); only positive contributions are summed (gray areas).

2005/06 through 2008/09 (Table 1), representing a sick leave rate of 1,952 (range: 1,595-2,607) per 100,000 persons employed. In the pandemic season, 84,359 GP-certified sick leaves with influenza were registered, an increase of $79 \%$ compared with the mean level in the previous seasons. The corresponding sick leave rate was 3,376 per 100,000 employed, a $73 \%$ increase compared to the average level in the non-pandemic seasons.

We estimated the number of sick leaves for other respiratory diseases with influenza as underlying cause using Poisson regression. In the model, $I L I^{15-64 y}$ (p<0.001) and $R S V$ (p<0.05) were significant predictor variables. We found an averxage of 22,150 (mean $95 \%$ $\mathrm{Cl}: 10,957-38,463)$ annual sick leaves associated with seasonal influenza (Table 1, Figure 2), corresponding to an average rate of 915 (mean $95 \% \mathrm{Cl}: 453-1,590$ ) sick leaves per 100,000 persons employed. The data suggest that influenza-related complications account for 9.2\% (range: $7.9-11.8 \%$ ) of sick leaves with other respiratory disease diagnoses. In the pandemic season, $24,371(95 \% \mathrm{Cl}: 13,531-37,427)$ sick leaves were found associated with influenza, indicating a slight increase 
A

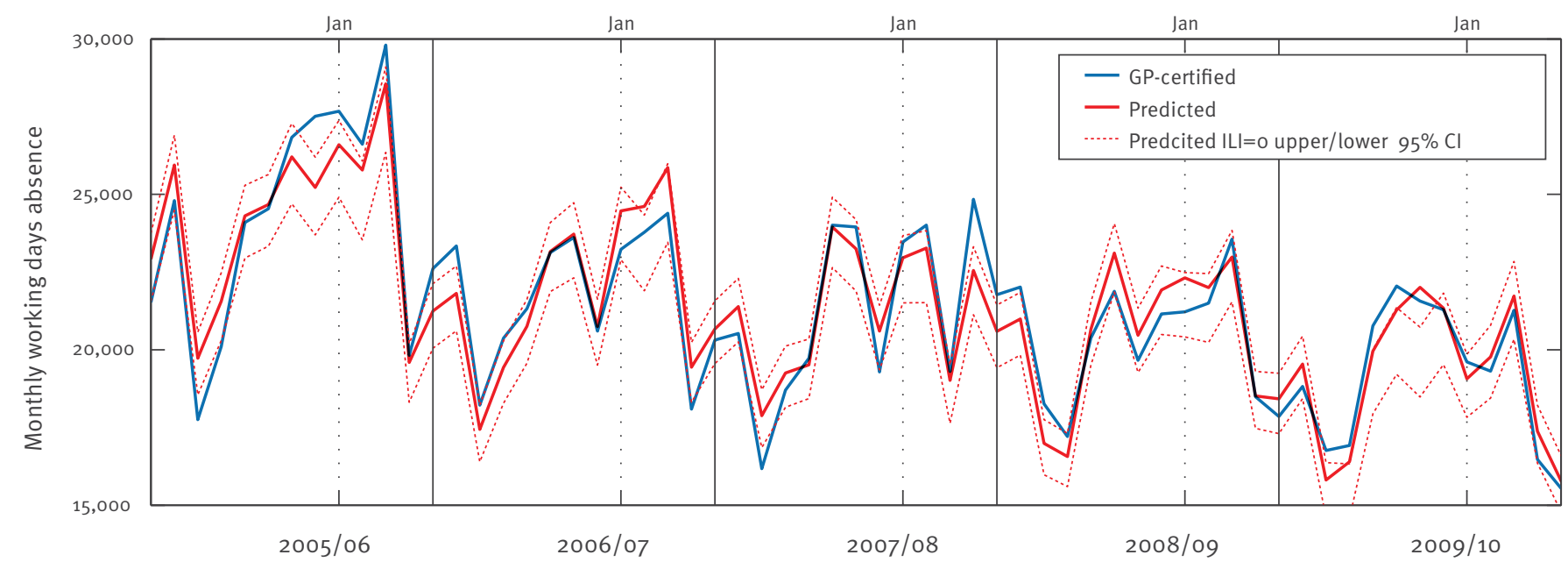

B

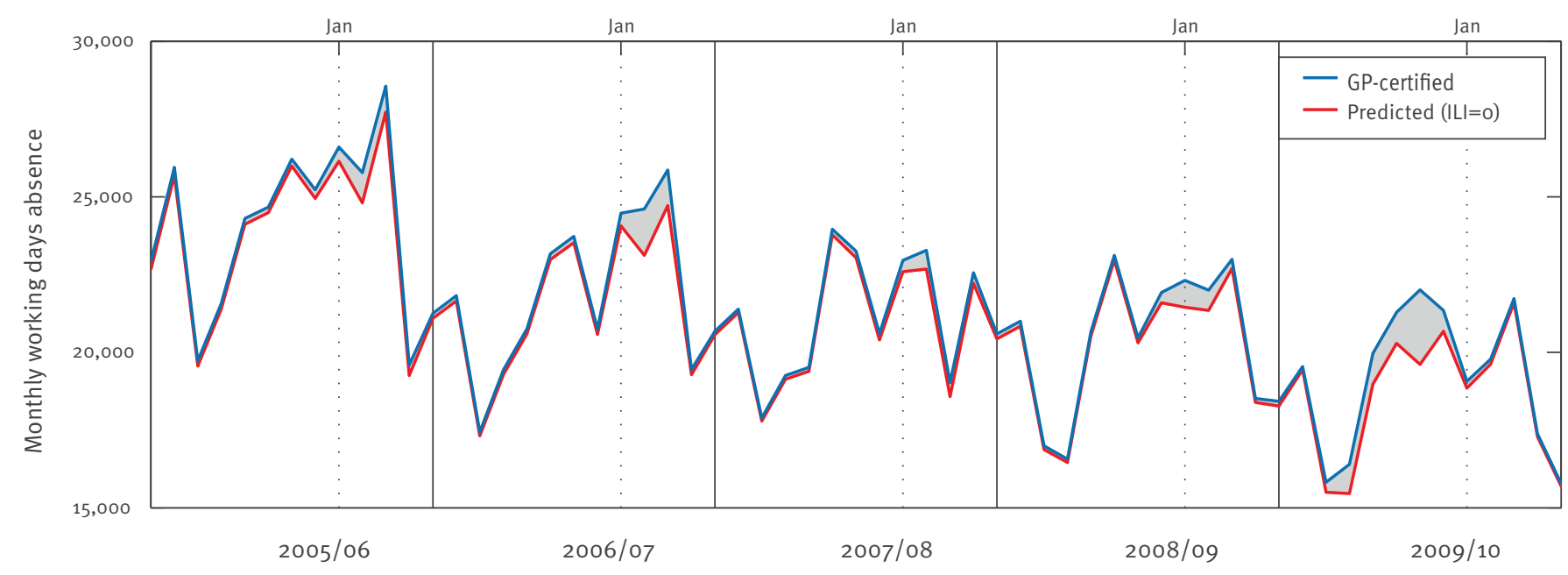

$\mathrm{CI}$ : confidence interval; GP: general practitioner; ILI: influenza-like illness.

A: Observed (blue) and predicted (red) monthly numbers of self-certified working days lost; upper and lower $95 \%$ confidence interval of prediction with $\mathrm{ILI}=0$ (dotted red lines).

B: Crude estimation calculated as the difference between observed and predicted (ILI=0), summing only positive contributions ( $\mathrm{gray}$ areas).

of $10 \%$ compared with the level typical for seasonal influenza. The sick leave rate was 975 ( $95 \% \mathrm{Cl}: 542-$ $1,498)$ per 100,000 employees and showed a similar trend, increasing by $7 \%$ compared with prior seasons.

Overall, we estimated a seasonal average of 69,396 (mean 95\% Cl: 58,203-85,709) influenza-associated sick leaves, indicating that 2,868 (mean $95 \% \mathrm{Cl}$ : $2,405-4,820$ ) per 100,000 persons employed obtain sick leave due to influenza annually. In the pandemic season the number of influenza-associated sick leaves was estimated at 108,730 (95\% Cl: $97,890-121,780)$ and was significantly increased compared with the previous years, while an estimated 4,352 $(95 \% \mathrm{Cl}$ : $3,918-7,728)$ per 100,000 persons employed went on sick leave with influenza-related illness.

We found large differences in the sick leave rates for seasonal influenza across the country: Health Region South had the highest sick leave rate with an average of 5,149 (mean $95 \% \mathrm{Cl}: 3,783-8,669$ ) per 100,000 persons employed in 2005/06-2008/09, 3.2 times the level of Health Region East, which had the lowest level estimated at 1,600 (mean $95 \% \mathrm{Cl}: 1,287-2,686$ ). 
During the pandemic season, influenza-diagnosed sick leaves increased by 70-90\% in Health Regions East, South and West compared with the levels typical for seasonal influenza. After adjusting for RSV, other respiratory sick leaves associated with influenza rose by factors of $10-20 \%$ in those regions. In Health Regions Mid and North, influenza sick leaves increased by 50-60\%, while respiratory sick leaves decreased by $15-20 \%$ compared with the seasonal mean.

We also tested a model on all short-term sick leaves (excluding all ICPC-2 R diagnoses), but no influenzaattributable excess sick leave was discovered, and these results are not presented.

\section{Self-certified and general practitioner-}

certified sick leave at Norway Post

\section{AS associated with influenza}

The total number of working days at Norway Post AS, i.e. the number of person-days after deduction of leaves (except sick leaves) and holidays, declined by $15 \%$ from 3.41 million in the $2005 / 06$ season to 2.91 million in the $2009 / 10$ season. In the study period, self-certified and GP-certified work loss comprised $1.3 \%$ and $8.0 \%$, respectively, of the total working days in the company.

Influenza-attributable work loss at Norway Post AS was estimated using Poisson regression. In the model for self-certified work absence, ILI ${ }^{15-64 y}$ (p<0.0001) was found a significant predictor variable, whereas Noro and $R S V$ were left out as neither was significant. The average estimated work loss due to seasonal influenza was 4,363 (mean 95\% Cl: 3,500-6,238) working days annually, $10.3 \%$ of the total load of self-certified absence in the company (Table $2 \mathrm{~A}$; Figure 3 ). The corresponding mean absence rate was 132 (mean 95\% $\mathrm{Cl}$ : 75-188) per 100,000 working days which was similar to the level obtained in the pandemic season of 127 (95\% Cl: 63-181) per 100,000 working days.

In the model for GP-certified sick leave, ILI $I^{15-64 y}$ was found significant $(\mathrm{p}=0.01)$ when excluding $R S V$, Noro and the interaction terms between ILI and the pandemic waves. The estimated influenza-associated work loss averaged at 7,295 (mean 95\% Cl: 1,702-17,204) working days per season, representing $2.7 \%$ of the total number of GP-certified working days lost (Table $2 \mathrm{~A}$, Figure 4). The corresponding rate of GP-certified absence was 219 (mean 95\% Cl: 51-514) per 100,000 working days for seasonal influenza. In the pandemic season, the estimated absence rate increased 1.5 -fold to 331 (95\% $\mathrm{Cl}$ : 113-675) per 100,000 working days.

\section{Influenza-attributable work absence to}

\section{care for sick children at Norway Post AS}

Between February 2008 and November 2010, female employees accounted for $43.3 \%$ of the total working days. The average age of the women was 47 years, versus 44 years among the men, and $54 \%$ of the women were aged 20-49 years compared to $61 \%$ of the men. The overall work absence to care for sick children comprised $0.27 \%$ of potential working days and was comparable among women (0.26\%) and men (0.29\%).

In the models predicting work absence of women and men due to parental care, $I L I^{0-14 y}$ ( $\left.\mathrm{p}<0.0001\right)$ was significant, while both $R S V$ and Noro were non-significant and were left out. In the $2008 / 09$ season, influenza-attributable work loss for sick children was estimated at 10 (95\% Cl: 2-27) and 13 (95\% Cl: 6-29) days per 100,000 working days for women and men, respectively. The results indicate that seasonal influenza accounts for approximately $4-5 \%$ of work absence related to care for sick children (Table 2B). Work absence increased significantly among women to 44 (95\% Cl: 30-63) per 100,000 working days and among men to 52 (95\% Cl: 33-75) per 100,000 working days during the pandemic season. Pandemic influenza accounted for $16-17 \%$ of the total work loss due to caring for sick children in the 2009/10 season.

\section{Total sick leave at Norway Post AS in}

2008/09-2009/10 associated with influenza

Overall, the work absence rate rose by a factor of 1.4 in the pandemic season, from an estimated 10,328 (95\% $\mathrm{Cl}$ : 4,339-24,326) working days lost in the 2008/09 season to $14,743(95 \% \mathrm{Cl}: 6,046-26,941)$ working days lost in the $2009 / 10$ season. In the pandemic season, self-certified work absence accounted for $25 \%$ of influenza-associated work loss, while $65 \%$ was GPcertified, and $10 \%$ was related to sick children. The corresponding figures for the $2008 / 09$ season were $36 \%$, $61 \%$ and $3 \%$, respectively.

\section{Discussion}

This study presents estimates of influenza-associated work absenteeism between 2005/06 and 2009/10 in Norway using both national and company-based data. Our results show that during an influenza season there are, on average, 47,200 influenza-diagnosed sick leaves, and a further 22,000 (mean 95\% Cl: 11,00038,500 ) sick leaves with other respiratory diseases are due to influenza-related complications. The results are consistent with the sick leave pattern over the seasons $1998 / 99$ to $2005 / 06$ that has been estimated at 48,300 influenza-diagnosed sick leaves and 11,000 influenzaattributable sick leaves with other respiratory illnesses [12]. In 2009/10, the number of sick leaves diagnosed for influenza almost doubled, while the number of influenza-attributable sick leaves diagnosed for other respiratory diseases was similar to the level seen in non-pandemic seasons. The average mean duration of GP-certified sick leave in Norway has been estimated at 7.2 days for influenza, and 10.6 days for other ICPC-2 $R$ diagnoses [12]. Using these figures, our analysis suggests that influenza accounts for 330,000 to 558,000 GP-certified working days lost per year. Assuming that the duration of sick leave was unchanged in the pandemic season, the corresponding estimate for the $2009 / 10$ season was 555,000 to 761,000 working days lost. 
Interestingly, we found large regional differences in sick leave rates associated with influenza, with similar trends during the pandemic and the non-pandemic seasons. This variation cannot be explained by regional deviation in influenza activity, i.e. by differences in ILI visits per capita in the regions. The reason for these large discrepancies may be regional habits of GPs using the influenza R8o code or other R codes, or may relate to differences in health seeking behaviour of patients in different regions, and needs to be explored further.

At Norway Post AS, a company that has an 'Inclusive Working Life' (IWL) agreement with full salary compensation during sickness for up to eight calendar days without a GP certificate, seasonal influenza accounted for a loss of $0.1-0.5 \%$ of the total working days. This proportion rose to $0.2-0.9 \%$ in the pandemic season. In comparison, a recent Canadian study found that seasonal influenza is responsible for a loss of $0.08 \%$ of potential hours worked annually with an elevated level of $0.2 \%$ in the $2009 / 10$ season [4]. In this study, the larger work loss in the pandemic season was associated with longer average duration of sick leaves, while the number of absent workers was similar to non-pandemic seasons.

The present use of Poisson regression is based on the correlation between ILI rates, laboratory-confirmed RSV and norovirus cases, and sick leave/work absence. The method is therefore sensitive to the quality of the surveillance data and temporal variations in the reported cases, and these limitations should be considered when interpreting the present findings. In particular, there may be time biases in the data collection and laboratory testing as well as in the GP-seeking behaviour during in the pandemic season.

Evaluation of the handling of the 2009 influenza $A\left(\mathrm{H}_{1} \mathrm{~N}_{1}\right)$ pandemic in Norway concluded that the surveillance of ILI provided a reliable representation of influenza activity, except at the beginning of the summer wave [19]. The summer wave coincided with an outbreak of human rhinovirus (HRV) in Norway, and circulation of HRV may have reduced the probability of influenza infection [2022]. The increase in ILI diagnoses during the summer may therefore initially have been caused by HRV, which is also supported by a Norwegian serological survey conducted in August 2009 showing low prevalence of antibodies reactive to the influenza $A\left(\mathrm{H}_{1} \mathrm{~N}_{1}\right)$ pdmo9 similar to the prevalence in sera collected in 2008 [23]. It is notable that $28 \%(24,092 / 84,359)$ of the influenzadiagnosed GP-certified sick leaves occurred from July to August before major circulation of the influenza $(A)$ $\mathrm{H}_{1} \mathrm{~N}_{1}$ pdmog virus was laboratory-confirmed. If these contributions are excluded from the estimates in Table 1 , the GP-certified sick leave rates during the pandemic was quite similar to the levels typical of seasonal epidemics. There also appeared to be an excess of influenza-associated sick leaves due to respiratory illness during the summer wave (see Figure 2).
From 28 April 2009, Norwegian public health authorities recommended that people with a suspected influenza illness should stay at home for seven days following the onset of symptoms [24], in line with the advice from the United States Centers for Disease Control and Prevention (CDC). On 18 November 2009, revised recommendations suggested a return to work no earlier than 24 hours after cessation of fever and general symptoms [25]. Ignoring all other factors, this may have increased the number of influenza-related working days lost per sick employee in 2009/10 compared to a normal season. In addition, employees in companies without an IWL agreement had an additional economic incentive to seek medical assistance during the pandemic because without a GP certificate, they were only allowed to stay absent from work for three days with full salary. However, at the beginning of the major pandemic wave (23 October 2009, Figure 1), the Norwegian government and social partners implemented a common policy to extend the self-certification period from three to eight days for non-IWL employees; the agreement remained active until 25 March 2010. In the official Norwegian review of the management of the pandemic it was argued that the seven-day rule may have led to unnecessarily high work absenteeism, in particular during the early phase of the pandemic [23].

Norway Post AS adhered to the national guidelines, which could have caused employees to take more time off work, thereby leading to higher self-certified work absence. However, the data are not suggestive of such a trend. Interestingly, only GP-certified work loss increased, while self-certified work absence remained at a level typical for seasonal influenza. One possible explanation for this finding could be that a larger proportion of employees with influenza symptoms consulted a GP due to awareness and fear of the ongoing pandemic, which is in line with the national data showing increased sick leave rates during the pandemic. However, to our knowledge there is no published literature on this subject. Our finding of a four-fold increase, compared with the 2008/09 epidemic, in influenzarelated absence of parents taking care of sick children, corroborates findings that the pandemic mainly affected children and young adults [26-28].

There are additional methodological limitations to the present study. Firstly, we only considered respiratory illnesses in the analysis of GP-certified sick leave. Therefore the excess sick leave observed here may underestimate the true absence burden related to influenza. Other diseases such as cerebrovascular disease or other chronic conditions could have been considered in the analyses, as has been done in studies of excess mortality [9], but their importance for sick leaves is currently not known. Secondly, influenza creates shortlived epidemics of six to eight weeks duration and our estimates would have improved if weekly information on work absence had been available from Norway Post AS. Thirdly, the laboratory data on RSV and norovirus 
infections are limited and may not fully represent the underlying epidemics. Fourthly, the analysis concerning GP-certified sick leave at Norway Post AS resulted in estimates with very wide confidence bounds and were less clear than the other analyses with respect to the impact of influenza activity, probably because the majority of GP-certified work absence is caused by long-term illnesses not related to influenza. More precise estimates for influenza could have been achieved if data on short-term work absence had been available. Finally, the findings related to Norway Post AS cannot necessarily be generalised to other types of work places, for instance in the healthcare sector and at schools.

In conclusion, we found higher GP-certified sick leave rates due to influenza in the pandemic season and higher absence rate for parents taking care of sick children at Norway Post AS, while self-certified sick leave in the company was similar to the levels in nonpandemic seasons. Fear of the pandemic, public health recommendations and extension of the duration of paid sick days are likely to have influenced work absence in the $2009 / 10$ season. The duration of paid sick days varies on the Norwegian labour market, which may affect absence rates and GP-seeking behaviour. More studies are needed to quantify the burden of self-certified sick leave, which comprises a substantial part of the economic burden of influenza to society.

\section{Acknowledgments}

Birgitte Freiesleben de Blasio and Yiting Xue were supported by The Research Council of Norway (Project: 166056/V50 and $177401 / V_{50}$ ). We thank Johan Swärd from Norway Post AS for providing data on work absence and helpful discussion, and Gabriel Ånestad from the Department of Virology, Division of Infectious Disease Control, Norwegian Institute of Public Health, for providing data on laboratory reports of RSV and norovirus. We are also grateful to Arna Desser for her assistance with proofreading.

\section{References}

1. Meltzer MI, Cox NJ, Fukuda K. The economic impact of pandemic influenza in the United States: priorities for intervention. Emerg Infect Dis. 1999;5(5):659-71.

2. Molinari NA, Ortega-Sanchez IR, Messonnier ML, Thompson WW, Wortley PM, Weintraub E et al. The annual impact of seasonal influenza in the US: measuring disease burden and costs. Vaccine. 2007;25(27):5086-96.

3. Keech M, Beardsworth P. The impact of influenza on working days lost: a review of the literature. Pharmacoeconomics. 2008;26(11):911-24.

4. Schanzer DL, Zheng H, Gilmore J. Statistical estimates of absenteeism attributable to seasonal and pandemic influenza from the Canadian Labour Force Survey. BMC Infect Dis. 2011;11:90.

5. Clark NM, Lynch JP, 3rd. Influenza: epidemiology, clinical features, therapy, and prevention. Semin Respir Crit Care Med. 2011;32(4):373-92.

6. Serfling R. Methods for current statistical analysis of excess pneumonia-influenza deaths. Public Health Rep. 1963;78(6):494-506.
7. Zucs P, Buchholz U, Haas W, Uphoff H. Influenza associated excess mortality in Germany, 1985-2001. Emerg Themes Epidemiol. 2005;2:6.

8. Simonsen L, Reichert TA, Viboud C, Blackwelder WC, Taylor RI, Miller MA. Impact of influenza vaccination on seasonal mortality in the US elderly population. Arch Intern Med. 2005;165(3):265-72.

9. Nunes B, Viboud C, Machado A, Ringholz C, Rebelo-deAndrade $\mathrm{H}$, Nogueira $\mathrm{P}$ et al. Excess mortality associated with influenza epidemics in Portugal, 1980 to 2004. PLoS One. 2011;6(6):e20661.

10. Gran JM, Iversen B, Hungnes O, Aalen OO. Estimating influenza-related excess mortality and reproduction numbers for seasonal influenza in Norway, 1975-2004. Epidemiol Infect. 2010;138(11):1559-68.

11. Thompson WW, Shay DK, Weintraub E, Brammer L, Bridges CB, Cox NJ et al. Influenza-associated hospitalizations in the United States. JAMA. 2004;292(11):1333-40.

12. Xue Y, Kristiansen IS, de Blasio BF. Modeling the cost of influenza: the impact of missing costs of unreported complications and sick leave. BMC Public Health. 2010;10:724.

13. Li S, Leader S. Economic burden and absenteeism from influenza-like illness in healthy households with children (5-17 years) in the US. Respir Med. 2007;101(6):1244-50.

14. Ånestad G, Vainio K, Hungnes O. Interference between outbreaks of epidemic viruses: additional Norwegian observations. Scand J Infect Dis. 2009;41(5):381-2.

15. Ånestad G, Vainio K, Hungnes O. Interference between outbreaks of epidemic viruses. Scand J Infect Dis. 2007;39(6-7):653-4.

16. The Norwegian Directorate of Health. International Classification of Primary Care 2nd Edition, electronic version [Accessed 3 Aug 2012]. Available from: http://kith.no

17. Statistics Norway. Employment and unemployment for persons aged 16-74 years [Accessed 15 Aug 2011]. Available from: http://www.ssb.no/

18. R Development Core Team. R: A Language and Environment for Statistical Computing. Vienna, Austria: R Foundation for Statistical Computing; 2008. Available from: http://www.rproject.org/

19. Linde A, Rotzen-Ostlund M, Zweygberg-Wirgart B, Rubinova S Brytting M. Does viral interference affect spread of influenza? Euro Surveill. 2009;14(40). Available from: http://www. eurosurveillance.org/ViewArticle.aspx?Articleld=19354

20. Casalegno JS, Ottmann M, Duchamp MB, Escuret V, Billaud $\mathrm{G}$, Frobert $\mathrm{E}$ et al. Rhinoviruses delayed the circulation of the pandemic influenza $A\left(\mathrm{H}_{1} \mathrm{~N}_{1}\right) 2009$ virus in France. Clin Microbiol Infect. 2010;16(4):326-9.

21. Ånestad G, Nordbo SA. Virus interference. Did rhinoviruses activity hamper the progress of the 2009 influenza $A\left(\mathrm{H}_{1} \mathrm{~N}_{1}\right)$ pandemic in Norway? Med Hypotheses. 2011;77(6):1132-4.

22. Waalen K, Kilander A, Dudman SG, Krogh GH, Aune T, Hungnes $O$. High prevalence of antibodies to the 2009 pandemic influenza $A\left(\mathrm{H}_{1} \mathrm{~N}_{1}\right)$ virus in the Norwegian population following a major epidemic and a large vaccination campaign in autumn 2009. Euro Surveill. 2010;15(31). Available from: http://www. eurosurveillance.org/ViewArticle.aspx?Articleld=19633

23. Direktoratet for samfunnssikkerhet og beredskap. Ny influenza $\mathrm{A}\left(\mathrm{H}_{1} \mathrm{~N}_{1}\right)$ 2009--gjennomgang av erfaringerne i Norge. [New influenza $A\left(\mathrm{H}_{1} \mathrm{~N}_{1}\right) 2009$ - a review of the Norwegian experience]. Direktoratet for samfunnssikkerhet og beredskap; 2010 Nov. Norwegian. Available from: http://www.dsb.no/ Global/Publikasjoner/2010/Rapporter/PandemiRapport.pdf

24. Norwegian Institute of Public Health. Statusrapport om ny influensa $\mathrm{A}\left(\mathrm{H}_{1} \mathrm{~N}_{1}\right)$, 7. januar 2010. [Status report on new influenza $A\left(\mathrm{H}_{1} \mathrm{~N}_{1}\right), 7$ January 2010]. [Accessed 10 Aug 2011]. Norwegian. Available from: http://www.fhi.no/dokumenter/.

25. Norwegian Institute of Public Health. Reviderte råd for anbefalinger om sykefraver ved influensaliknende sykdom. [Revised advise for recommendations on absenteeism due to influenza-like illness]. [Accessed 18 Nov 2009]. Norwegian. Available from: http://www.fhi.no/

26. De Blasio BF, Iversen BG, Scalia Tomba G. Effect of vaccines and antivirals during the major $2009 \mathrm{~A}\left(\mathrm{H}_{1} \mathrm{~N}_{1}\right)$ pandemic wave in Norway--and the influence of vaccination timing PloS ONE. 2012; 7(1): e30018.

27. Miller E, Hoschler K, Hardelid P, Stanford E, Andrews N, Zambon M. Incidence of 2009 pandemic influenza $\mathrm{A} \mathrm{H}_{1} \mathrm{~N}_{1}$ infection in England: a cross-sectional serological study. Lancet. 2010;375(9720):1100-8.

28. Yang Y, Sugimoto JD, Halloran ME, Basta NE, Chao DL, Matrajt L et al. The transmissibility and control of pandemic influenza A $\left(\mathrm{H}_{1} \mathrm{~N}_{1}\right)$ virus. Science. 2009;326(5953):729-33. 